\title{
Energy Dependence of Angular Momentum Capture States in Charge Exchange Collisions between Slow Highly Charged Argon Ions and Argon Neutrals
}

\author{
F.I. Allen, ${ }^{*}$ C. Biedermann, R. Radtke, and G. Fussmann \\ Lehrstuhl für Plasmaphysik, Institut für Physik der Humboldt-Universität zu Berlin, \\ Newtonstraße 15, 12489 Berlin and Max-Planck-Institut für Plasmaphysik, \\ EURATOM Association, 17491 Greifswald, Germany \\ S. Fritzsche \\ Gesellschaft für Schwerionenforschung, 64291 Darmstadt and \\ Max-Planck-Institut für Kernphysik, 69029 Heidelberg, Germany
}

(Dated: November 6, 2008)

\begin{abstract}
$K$-shell x-ray emission measurements of electron capture into Rydberg states of $\mathrm{Ar}^{17+}$ and $\mathrm{Ar}^{18+}$ ions are carried out as a function of collision energy in order to investigate the energy dependence of angular momentum capture states in slow charge exchange collisions. The highly charged ions are produced using an Electron Beam Ion Trap and extracted onto an external argon gas target. A retardation assembly in the beamline allows projectile energies from $\sim 2 \mathrm{keV} / \mathrm{amu}$ down to $\sim 5 \mathrm{eV} / \mathrm{amu}$ to be selected. For decreasing collision energies a shift to electron capture into low orbital angular momentum capture states is observed. Comparative $K$-shell x-ray emission measurements of electron capture by $\mathrm{Ar}^{17+}$ and $\mathrm{Ar}^{18+}$ ions from background gas in the trap are also presented. The results of the trapping experiments are in discrepancy with those obtained using extracted ions and possible explanations are discussed.
\end{abstract}

PACS numbers: 34.70.+e, 32.30.Rj, 32.70.Fw, 52.70.La

\section{INTRODUCTION}

Charge exchange between a highly charged ion (HCI) and a gas neutral is the process by which one or more electrons are captured from the neutral into Rydberg states of the HCI. In low energy collisions, where the projectile velocity is of the order of the orbital velocity of the target electron, charge exchange is the dominant interaction which occurs [1]. Subsequent to the interaction the excited HCI stabilizes via a combination of radiative decay and autoionization. Studies of charge exchange are important for a fundamental understanding of atomic processes and for applications such as spectral diagnostics of fusion plasmas heated by neutral beam injection [2] and the determination of ion storage times in ion traps and storage rings [3]. Further impetus to this area of research stems from the discovery of x-ray emission from comets by Lisse et al. in 1996 [4], for which charge exchange between HCIs in the solar wind and neutrals in the coma of the comet has been established as the main emission mechanism [5].

An analytical method which is widely used to describe the capture process in slow collisions is the classical overthe-barrier $(\mathrm{COB})$ model $[6,7]$. It is based on a consideration of the electrostatic potential experienced by the active electron during transfer. The principal quantum number of the dominant capture state, $n_{c}$, predicted by the model is given by:

\footnotetext{
*FIAllen@lbl.gov
}

$$
n_{c} \approx \frac{q^{3 / 4}}{\sqrt{I_{P} / 13.6}}
$$

where $q$ is the charge state of the HCI and $I_{P}$ is the ionization potential (in electronvolts) of the target. In low energy collisions $n_{c}$ is virtually independent of collision energy $[1,8]$ and the range of $n_{c}$ states which becomes populated is very narrow, i.e. $\Delta n_{c} \approx \pm 1$ [9].

As the COB model is essentially a static treatment, a similar estimate of the orbital angular momentum capture state, $\ell_{c}$, cannot be directly inferred. In high energy charge exchange collisions electrons populate $\ell_{c}$ states statistically, whereas in the low energy regime deviation from a statistical population occurs [8]. Assuming that the orbital angular momentum of the captured electron is given by the product of impact parameter and velocity, then for slower collisions capture into lower $\ell_{c}$ states is expected. Theoretical and experimental work in this area is limited, since studies have mainly focused on higher energy collisions of relevance to high temperature laboratory plasmas. However, for the interpretation of $\mathrm{x}$ ray emission from comets, for example, where the solar wind ions can be decelerated to $50 \mathrm{eV} / \mathrm{amu}$ and below $(\lesssim 100 \mathrm{~km} / \mathrm{s})$, a need for low energy data has arisen. In fact, measurements of the morphology of cometary emission have shown that up to $50 \%$ of the radiation might originate from inside the bow shock, near the comet nucleus where the HCI velocity is the lowest [10].

The radiative cascade of a captured electron to the ground state is characteristic of the initial capture state. Thus due to the energy dependence of $\ell_{c}$ it is proposed 
that the cometary spectra could be used to probe the dynamics of the solar wind and ultimately to monitor space weather, without the need for heliospheric spacecraft [11]. In the present work the effect of collision energy on $\ell_{c}$ is investigated by recording the $K$-shell x-ray emission spectra following electron capture by $\mathrm{Ar}^{17+}$ and $\mathrm{Ar}^{18+}$ ions extracted from an Electron Beam Ion Trap (EBIT) onto an argon gas target. The extraction beamline incorporates a retardation assembly, allowing experiments using HCIs with energies of the order of $\mathrm{keV} / \mathrm{amu}$ down to a few eV/amu to be carried out. X-ray emission measurements of the charge exchange of HCIs interacting with background gas in the trap have also been implemented, where ion energies are $\sim 10 \mathrm{eV} / \mathrm{amu}$.

Similar work is conducted by the EBIT groups at the National Institute of Standards and Technology (NIST) and Lawrence Livermore National Laboratory (LLNL), in the first case using extracted HCIs, though without a setup for deceleration [12], and in the second case solely by probing the HCIs in the trap [13]. The results presented in the current work therefore constitute the first detailed survey of charge exchange emission for a range of collision energies in the low energy regime. The trend observed indicates that for collision energies decreasing below $100 \mathrm{eV} / \mathrm{amu}$, deviation from a statistical population of states and preferential capture into low $\ell_{c}$ states occurs. This finds qualitative agreement with the results of Classical Trajectory Monte Carlo (CTMC) calculations [13], although this agreement may be fortuitous, as will be discussed. A discrepancy between the charge exchange spectra measured for the ions in the trap compared with those obtained at the external target is revealed and possible explanations are considered.

\section{EXPERIMENTAL METHOD}

The HCIs were produced nearly at rest using the Berlin EBIT, which is described in detail in [14], via successive impact ionization of an injected gas by a monoenergetic electron beam. Radial confinement of the ions in the trap is primarily determined by the space charge of the electron beam, which is highly compressed by the $3 \mathrm{~T}$ magnetic field generated using a pair of Helmoltz coils. Axial confinement results from the potential well created by the voltages on three drift tube electrodes.

\section{A. Extraction Experiments}

For the experiments with an external gas target, argon gas was injected into the EBIT at a pressure of $10^{-5} \mathrm{~Pa}$ and the HCIs generated were extracted in pulse mode at a rate of one ion bunch per second. The electron beam of the EBIT was set to a current of $120 \mathrm{~mA}$ and an axial trap depth of $100 \mathrm{~V}$ was implemented. During the breeding phase of each cycle, lasting $0.9 \mathrm{~s}$, the acceleration potential of the electrons into the trap region was set to $10.1 \mathrm{kV}$. This maximizes the yield of $\mathrm{Ar}^{17+}$ and $\mathrm{Ar}^{18+}$ ions. In the extraction phase the potential was lowered to give an extraction potential of $5.3 \mathrm{kV}$, which is more ideally suited to the beamline elements. The ion pulses were $\sim 30 \mathrm{~ms}$ in duration.

The HCIs were transported through the extraction beamline, which has been described in more detail in [15], selected according to their mass-to-charge ratio using a Wien filter and then directed onto the gas target. Just prior to the target area is a retardation assembly consisting of a series of parallel grids which are biased to give a uniform repelling field. The voltage on the last grid determines the final kinetic energy of the ions which then pass through a field-free drift region to the gas target. Experiments were carried out for projectile energies down to $10 q \mathrm{eV}$. The final energy was measured using a retarding field technique, as described in [15].

Argon gas was injected into the target region at $90^{\circ}$ to the ion beam axis using a pulsed supersonic valve with a repetition rate of $1 \mathrm{~Hz}$ and a pulse length of $60 \mu \mathrm{s}$. The gas pulses were synchronized to coincide with the arrival of ion bunches from the EBIT and the density of gas at the target is estimated at $10^{12} \mathrm{~cm}^{-3}$. The $\mathrm{x}$-ray emission resulting from charge exchange between the HCIs and argon neutrals was recorded using a thermoelectricallycooled silicon solid state detector mounted $44 \mathrm{~mm}$ from the target center, giving a solid angle for photon detection of $2.6 \cdot 10^{-3} \mathrm{sr}$. At $5.9 \mathrm{keV}$ the detector has a full width at half maximum (FWHM) peak resolution of $168 \mathrm{eV}$.

\section{B. Magnetic Trapping Experiments}

Charge exchange of HCIs in the trap was investigated by operating the EBIT in magnetic trapping mode. This method, introduced by Beiersdorfer et al. [16], involves periodically breeding HCIs in the trap and then switching the electron beam off to give phases when the radial confinement of ions only results from the magnetic field of the Helmholtz coils. In this way the emission resulting from charge exchange is unmasked from that due to electron-ion interactions, which dominate when the electron beam is present.

As in the extraction experiments, argon gas was injected into the EBIT at a pressure of $10^{-5} \mathrm{~Pa}$. The density of neutrals in the trap acting as partners for charge exchange is estimated from the confinement time and the theoretical charge exchange cross sections at $10^{6} \mathrm{~cm}^{-3}$. Rapid switching of the electron beam was achieved by controlling the anode voltage of the electron gun using a function generator in combination with a high voltage amplifier. In each switching cycle the electron beam was turned on, within $8 \mathrm{~ms}$, to the required ionization potential to give $1 \mathrm{~s}$ of breeding after which it was turned off, within $4 \mathrm{~ms}$, to give $0.8 \mathrm{~s}$ of magnetic trapping for the charge exchange experiment. At the end of each cycle the trap was briefly opened to expulse all ions and prevent 
the accumulation of background heavy ions.

In the breeding phase electron beam currents of $70 \mathrm{~mA}$ and $100 \mathrm{~mA}$, for the experiments with $\mathrm{Ar}^{17+}$ and $\mathrm{Ar}^{18+}$ ions, respectively, were selected and axial trap depths of 30,100 and $700 \mathrm{~V}$ were applied. The acceleration potential of the electron beam was set to $4.3 \mathrm{kV}$ in the $\mathrm{Ar}^{17+}$ experiments, which is just below the ionization threshold for forming $\mathrm{Ar}^{18+}$, and to $10.1 \mathrm{kV}$ in the $\mathrm{Ar}^{17+}$ experiments. The contributions from $\mathrm{Ar}^{17+}$ ions which are inevitably also present in the trap during the $\mathrm{Ar}^{18+}$ experiments were subtracted out of the x-ray spectra, as will be discussed.

The x-ray emission was recorded as a function of time using a windowless liquid nitrogen-cooled germanium solid state detector mounted $85 \mathrm{~mm}$ from the trap axis, giving a solid angle for photon detection of $4.2 \cdot 10^{-3} \mathrm{sr}$. At $5.9 \mathrm{keV}$ a FWHM peak resolution of $136 \mathrm{eV}$ is measured.

\section{SPECTRA AND DATA ANALYSIS}

For electron capture by $\mathrm{Ar}^{17+}$ and $\mathrm{Ar}^{18+}$ ions from argon neutrals, Equation 1 gives a COB prediction of capture into $n_{c}=8$. It follows that radiative cascades from the $n_{c} \ell_{c}$ states $8 s$ to $8 j$ are then possible. In order to facilitate the interpretation of the charge exchange spectra measured in the experiments, the cascade paths from these various capture states have been simulated. The input data for the calculations, comprising values for the energy levels and transition probabilities for hydrogenlike and helium-like argon ions, were obtained using the Relativistic Atomic Transition and Ionization Properties (RATIP) code $[17,18]$. In Figure 1 the $K$-shell emission spectra simulated for electron capture into $\mathrm{Ar}^{18+}$, with a line broadening of $160 \mathrm{eV}$, are presented. It can be seen that for cascades from different $8 \ell_{c}$ states the emission varies significantly. For $\mathrm{Ar}^{17+}$ charge exchange the x-ray spectra are less sensitive to capture state due to the presence of the unpaired electron in the $K$-shell, which reduces the number of transition paths available to a cascading electron.

\section{A. Extraction Spectra}

In the extraction experiments the Wien filter was used to select the particular charge state to be investigated. However, as a result of charge exchange in the beamline subsequent to charge state selection, a proportion of lower charge state ions still arrived at the target. For the $\mathrm{Ar}^{17+}$ spectra this effect is not important, because electron capture into argon ions with $q \leq 16$ does not result in $K$-shell emission. For the $\mathrm{Ar}^{18+}$ measurements, however, emission associated with $\mathrm{Ar}^{17+}$ ions which arrived at the target was present in the spectra recorded. In order to isolate the emission resulting from electron capture into $\mathrm{Ar}^{18+}$ in a given measurement, the lower energy $K \alpha$
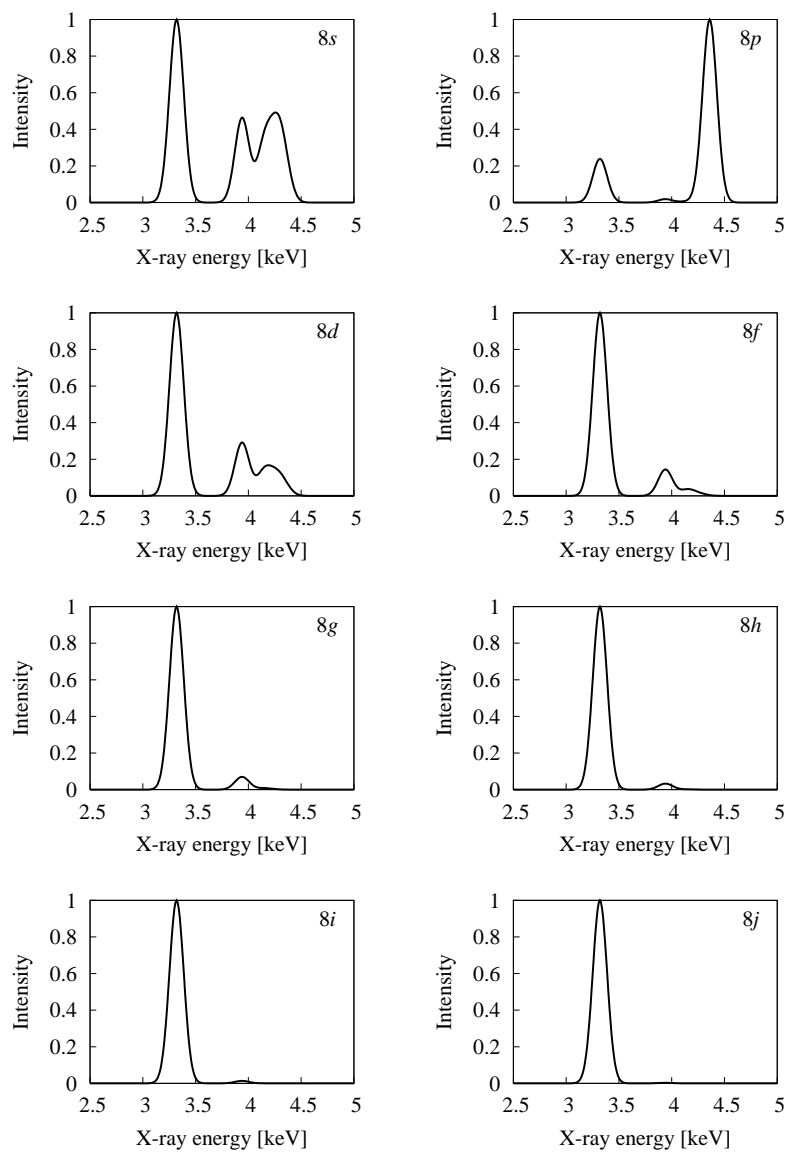

FIG. 1: Normalized x-ray emission spectra simulated for electron capture by $\mathrm{Ar}^{18+}$ into the states $8 s$ to $8 j$, with a line broadening of $160 \mathrm{eV}$.

peak from $\mathrm{Ar}^{17+}$ charge exchange was used to generate a normalized spectrum from an $\mathrm{Ar}^{17+}$ measurement taken at a similar energy, and this was then subtracted out.

Selected results from the experiments using beams of HCIs extracted onto an external argon gas target are presented in Figure 2; Figures 2(a) and 2(b) show the charge exchange spectra for $50 q \mathrm{eV}$ and $515 q \mathrm{eV} \mathrm{Ar}^{17+}$ ions, and Figures 2(c) and 2(d) show those obtained for $40 q \mathrm{eV}$ and $485 q \mathrm{eV} \mathrm{Ar}{ }^{18+}$ ions, respectively. The experimental data are plotted in gray and for reference the positions of the various $K$-shell x-rays together with the series limit for each ion charge state are marked in the figures on the right. Fits to the data are represented by black curves and comprise three Gaussian distributions corresponding to $n=2 \rightarrow 1, n=3 \rightarrow 1$ and $n \geq 4 \rightarrow 1$ transitions.

The relative intensities of the $\mathrm{x}$-ray peaks in the $\mathrm{Ar}^{17+}$ spectra of Figures 2(a) and 2(b) are very similar. Furthermore, on comparing these results with the additional $\mathrm{Ar}^{17+}$ spectra recorded, the $\mathrm{Ar}^{17+}$ spectral shapes are found to vary very little over the collision energy range studied. The spectra obtained closely resemble the results of EBIT experiments carried out by Tawara et al. $[12,19]$, which used beams of $\mathrm{Ar}^{17+}$ ions extracted onto 


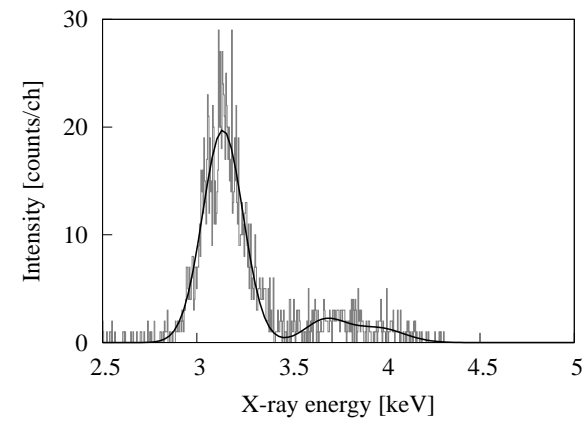

(a) $50 q \mathrm{eV} \mathrm{Ar}{ }^{17+}$

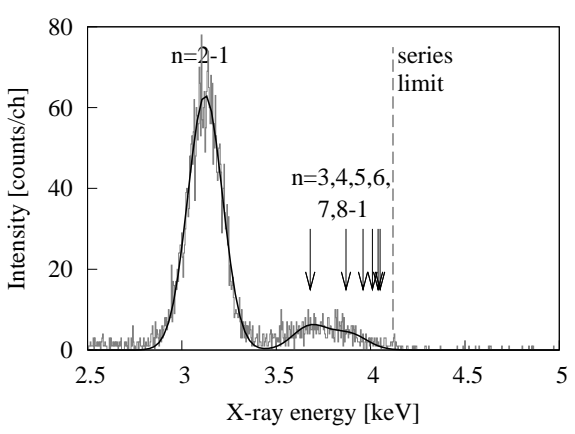

(b) $515 q \mathrm{eV} \mathrm{Ar}{ }^{17+}$

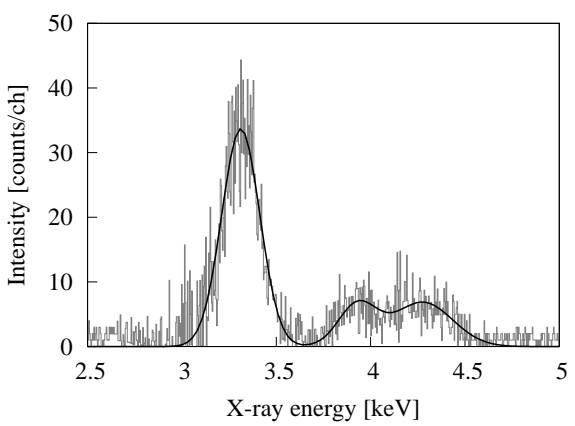

(c) $40 q \mathrm{eV} \mathrm{Ar}{ }^{18+}$

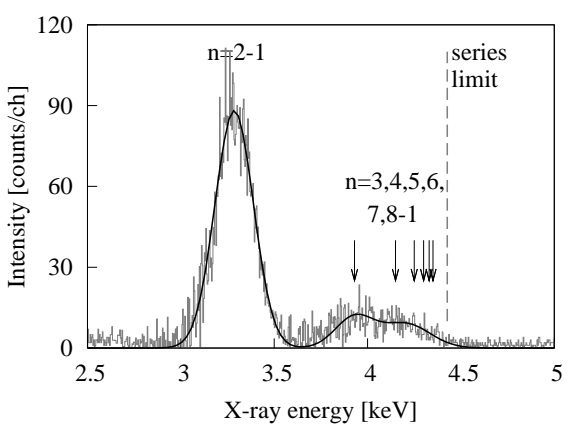

(d) $485 q \mathrm{eV} \mathrm{Ar}{ }^{18+}$

FIG. 2: X-ray emission spectra for charge exchange of $\mathrm{Ar}^{17+}$ and $\mathrm{Ar}^{18+}$ ions with an argon gas target for a range of projectile energies. Experimental data are plotted in gray and black curves represent the sum of Gaussian fits. an argon gas target at energies of $3 q \mathrm{keV}$ and $8 q \mathrm{keV}$, respectively.

In contrast, in the case of the results for $\mathrm{Ar}^{18+}$ charge exchange, a variation in spectral shape versus collision energy is observed. The emission spectra for $485 q \mathrm{eV}$ $\mathrm{Ar}^{18+}$ ions (Figure 2(d)) and non-retarded $\mathrm{Ar}^{18+}$ ions (not shown) resemble a spectrum obtained at NIST by Tawara et al. using beams of $8 q \mathrm{keV} \mathrm{Ar}^{18+}$ ions [12], whereas the spectra for $\mathrm{Ar}^{18+}$ ions with energies of $40 q \mathrm{eV}$ (Figure 2(c)) and lower show an enhancement of the higher energy $n \geq 3 \rightarrow 1$ emission.

\section{B. Magnetic Trapping Spectra}

For the analysis of charge exchange emission from HCIs in the EBIT in magnetic trapping mode, only those $\mathrm{x}$ ray counts recorded in each cycle $10 \mathrm{~ms}$ after the electron beam was switched off are considered. This is well above the electron beam ramp down time, which ensures that the x-ray spectra are completely free of photons emitted as a result of HCI interactions with the electron beam. The results obtained for $\mathrm{Ar}^{17+}$ and $\mathrm{Ar}^{18+}$ ions, for a trap depth of $100 \mathrm{~V}$, are presented in Figure 3. The experimental data are plotted in gray. Note that as for the analysis of the extraction spectra, emission components due to the presence of $\mathrm{Ar}^{17+}$ ions have been subtracted from the $\mathrm{Ar}^{18+}$ plot. Fits to the data are represented by black curves. In Figure 3(a) the fit again corresponds to three Gaussian distributions for $n=2 \rightarrow 1, n=3 \rightarrow 1$ and $n \geq 4 \rightarrow 1$ transitions, whereas in Figure 3(b) four Gaussian distributions are fitted, corresponding to $n=2 \rightarrow 1$, $n=3 \rightarrow 1, n=4 \rightarrow 1$ and $n \geq 5 \rightarrow 1$ transitions.

The $\mathrm{Ar}^{17+}$ emission spectrum of Figure 3(a) closely resembles the $\mathrm{Ar}^{17+}$ spectra recorded in the extraction experiments. The spectra recorded using 30 and $700 \mathrm{~V}$ trap depths are hardly distinguishable from the $100 \mathrm{~V}$ measurement shown, and the results are also very similar to an $\mathrm{Ar}^{17+}$ magnetic trapping spectrum obtained by Beiersdorfer et al. at LLNL, for which a trap depth of $300 \mathrm{~V}$ was implemented [13].

In the $\mathrm{Ar}^{18+}$ spectrum of Figure $3(\mathrm{~b})$, the relative intensity of the $n \geq 3 \rightarrow 1$ emission band is significantly greater than in its $\mathrm{Ar}^{17+}$ magnetic trapping as well as extraction counterparts. The emission associated with the closely-lying $n \geq 5 \rightarrow 1$ transitions, in particular, is very prominent. This indicates a large proportion of capture into $p$ states, as exemplified by the simulated spectra presented in Figure 1.

The shape of the $100 \mathrm{~V}$ magnetic trapping $\mathrm{Ar}^{18+}$ spectrum likens the result of a similar measurement made at the LLNL EBIT for a trap depth of $300 \mathrm{~V}$ [13], as well the further spectra recorded in the present work for the trap depths 30 and $700 \mathrm{~V}$. It appears that the centroid of the highest energy peak is $\sim 20 \mathrm{eV}$ higher than the theoretical energy of the $8 p \rightarrow 1 s$ transition. This suggests that electron capture might predominantly occur one or two shells higher than the $n_{c}=8$ prediction from the 


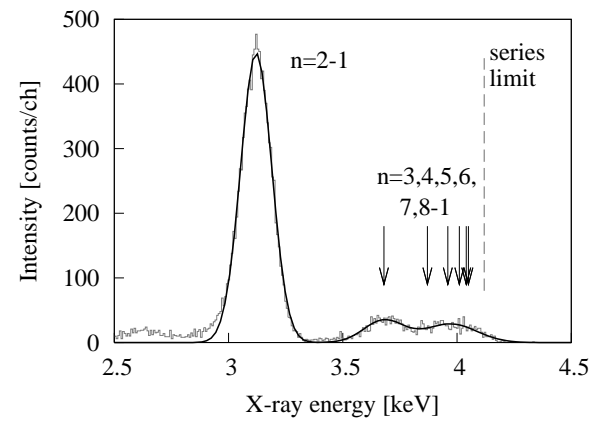

(a) $100 \mathrm{~V}$ trap $\mathrm{Ar}^{17+}$

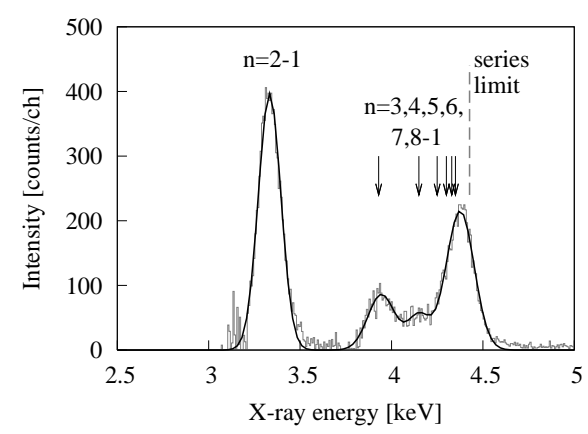

(b) $100 \mathrm{~V}$ trap $\mathrm{Ar}^{18+}$

FIG. 3: X-ray emission spectra for charge exchange of $\mathrm{Ar}^{17+}$ and $\mathrm{Ar}^{18+}$ ions with background gas in the EBIT operated in magnetic trapping mode. Experimental data are plotted in gray and black curves represent the sum of Gaussian fits.

COB model. Indeed, slight underestimation of $n_{c}$ by the COB model has been reported elsewhere [20]. The magnetic trapping $\mathrm{Ar}^{18+}$ charge exchange spectra measured at LLNL suggest that $n_{c}=9$ dominates $[13,21]$.

The principal charge exchange partner in the trap is the argon gas which is continuously injected into the chamber, but electron capture from other background gas species, mainly nitrogen and oxygen, can also occur. The ionization potential of molecular nitrogen $(15.6 \mathrm{eV})$ is very close to that of argon $(15.8 \mathrm{eV})$, thus according to Equation 1 a shift in $n_{c}$ is not expected. However, in the case of molecular oxygen (ionization potential $12.1 \mathrm{eV}$ ), $n_{c}=9$ is predicted. Therefore the aforementioned emission at energies higher than the $8 p \rightarrow 1 s$ transition could have also resulted from charge exchange with oxygen in the trap. Nevertheless the distribution of $\ell_{c}$ states should be very similar and indeed experiments conducted elsewhere, investigating the target dependence of charge exchange $\mathrm{x}$-ray emission, indicate that the ionization potentials of argon and oxygen are still close enough not to significantly alter the relative intensities of the radiative emission peaks observed [22].

\section{Comparison of Spectra via Hardness Ratios}

Comparison of the various x-ray emission spectra recorded in the extraction and magnetic trapping experiments is achieved by calculating hardness ratios, $\mathcal{H}$, defined as the intensity ratio of all $n \geq 3 \rightarrow 1$ to $n=2 \rightarrow 1$ transitions, i.e:

$$
\mathcal{H}=\frac{\sum_{n=3}^{n_{c}} I_{n \rightarrow 1}}{I_{2 \rightarrow 1}}
$$

The result is shown in Figure 4, a plot of hardness ratio versus center-of-mass collision energy. Black symbols mark the data obtained from the spectra measured in this work, while those obtained elsewhere are marked in gray.

The results from the extraction experiments, plotted using solid black triangles and circles to denote the hardness ratios for $\mathrm{Ar}^{17+}$ and $\mathrm{Ar}^{18+}$ charge exchange, respectively, are connected with black lines to guide the eye. The hardness ratios presented are the result of a more thorough data analysis than those given in [23]. Hence some of the values have been modified, in particular the hardness ratio for the slowest $\mathrm{Ar}^{18+}$ ions implemented in the present study. The center-of-mass collision energy is given in electronvolts per atomic mass unit, calculated from the projectile energies assuming a reduced mass of $m_{A r} / 2$. The error bars are calculated from the FWHM values obtained from the reverse bias measurements detailed in [15]. An additional scale showing the relative collision velocity in atomic units, i.e. as a fraction of the Bohr velocity, is shown. The error bars for the hardness ratios are Gaussian errors determined from the uncertainty in the fitted peak areas.

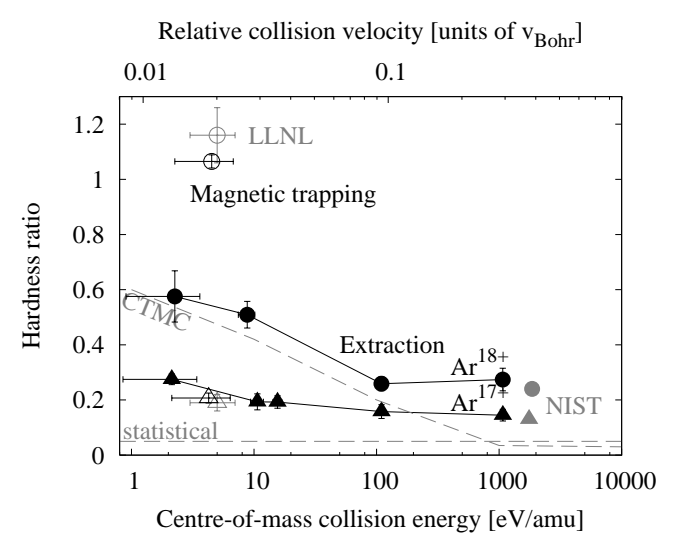

FIG. 4: Dependence of hardness ratio on center-of-mass collision energy for charge exchange of $\mathrm{Ar}^{17+}$ and $\mathrm{Ar}^{18+}$ with argon neutrals. Solid and hollow black symbols represent the results of the extraction and magnetic trapping experiments, respectively. Gray symbols show extraction and magnetic trapping measurements made by NIST [12] and LLNL [13]. CTMC results for single electron capture into $\mathrm{Ar}^{18+}$ from a hydrogen target [13] and the hardness ratio predicted on the basis of statistical assumptions are marked with dashed lines. 
Hardness ratios for $\mathrm{Ar}^{17+}$ and $\mathrm{Ar}^{18+}$ charge exchange measured in the magnetic trapping experiments for a trap depth of $100 \mathrm{~V}$ are plotted using a hollow black triangle and circle, respectively. The temperature of HCIs in an EBIT is a function of the axial trapping potential, and is estimated here using the empirical relation:

$$
T_{\text {ion }}=0.2 q V_{\text {trap }},
$$

where $V_{\text {trap }}$ is the applied axial trapping potential and $q$ is the charge state of the HCI [24]. In the figure the error assumed for the temperature estimates is $50 \%$ and as for the extraction data, center-of-mass collision energies are plotted. With respect to the hardness ratios published in [23], the values plotted here are shifted slightly. This is because it has since been found that the hardness ratios measured can vary slightly according to the length of the magnetic trapping cycle. Therefore care was taken to ensure that only spectra of equal magnetic trapping phase durations were compared. Data from the LLNL magnetic trapping experiments using a trap depth of $300 \mathrm{~V}$ [13] are plotted in the figure using hollow gray symbols. The data points are taken from Figure 5 of the cited paper, rather than from Figure 1 where the hardness ratio for electron capture into $\mathrm{Ar}^{18+}$ appears to correspond to the intensity ratio of $n \geq 4 \rightarrow 1$ to $n=2 \rightarrow 1$ emission.

Based on a statistical population of states in a given shell $n_{c}$, which is the scenario relevant to high energy charge exchange collisions, the hardness ratio expected can be calculated from the fractional population of the $n_{c} p$ state. Thus for high energy $\mathrm{Ar}^{17+, 18+}$ charge exchange, assuming $n_{c}=8$, a hardness ratio of $\sim 0.05$ is inferred. This is marked in Figure 4 using a horizontal dashed line. Comparing with the hardness ratios obtained in the extraction experiments, however, a significant variation with collision energy, particularly in the case of $\mathrm{Ar}^{18+}$ charge exchange, is observed. Hence it can be concluded that the collisions investigated were slow enough for a non-statistical population of $\ell_{c}$ states to occur. Furthermore, the trend is for hardness ratio to increase with decreasing projectile velocity, indicating that in lower energy collisions capture into lower $\ell_{c}$ states is favored, i.e. for lower collision velocities there is a higher proportion of capture into $n_{c} p$ states, resulting in the direct $n_{c} p \rightarrow 1 s$ cascade transitions which give the larger hardness ratio measured.

A similar theoretical trend has been found by Beiersdorfer et al. by implementing a CTMC method to simulate electron capture into slow $\mathrm{Ar}^{18+}$ ions from hydrogen neutrals and then calculating the resulting radiative cascades using a decay matrix for hydrogenic ions [13]. The results from these simulations are represented in Figure 4 by a dashed gray line labeled 'CTMC'. Although there is qualitative agreement between the experimental and CTMC data for $\mathrm{Ar}^{18+}$, this may be fortuitous for the following reasons. Firstly, the CTMC method does not include molecular effects, thus it cannot accurately describe electron capture in low energy collisions. In the case of highly charged projectiles, however, the impor- tance of this molecular behavior is somewhat diminished [25]. Secondly, the CTMC data from [13] are for single electron capture, whereas in slow HCI-atom collisions the cross sections for multiple electron capture are in fact significant [26]. Indeed it has been shown that multiple electron capture plays an important role in the shaping of charge exchange emission spectra [12, 27]. However, the overall magnitude of the hardness ratio might not be changed, since multiple capture events mainly rearrange the $n p \rightarrow 1 s$ x-ray emission to lower $n$ levels.

The experimental hardness ratios for $\mathrm{Ar}^{18+}$ charge exchange are consistently higher than the corresponding values for $\mathrm{Ar}^{17+}$ and the increase in ratio with decreasing collision velocity is more marked for $\mathrm{Ar}^{18+}$. This is supported by the hardness ratios inferred from the simulated spectra, where it is seen that, in general, the $\mathrm{Ar}^{18+}$ hardness ratios for capture into the various $\ell_{c}$ states are higher than their $\mathrm{Ar}^{17+}$ counterparts, especially for low $\ell_{c}$, and that the hardness ratios for $\mathrm{Ar}^{18+}$ vary over a wider range. A larger hardness ratio for $\mathrm{Ar}^{18+}$ than for $\mathrm{Ar}^{17+}$ charge exchange is also inferred from measurements made by the NIST EBIT group in similar experiments using non-retarded $\mathrm{Ar}^{17+}$ and $\mathrm{Ar}^{18+}$ ions incident on an external argon gas target [12]. The NIST data points are plotted in the figure using solid gray triangles and circles, respectively, and lie in the range of the data obtained in the present work using non-retarded ions.

\section{DISCUSSION}

On inspection of Figure 4 it can be seen that the hardness ratios from the extraction experiments with $\mathrm{Ar}^{18+}$ ions are at odds with the values for charge exchange by $\mathrm{Ar}^{18+}$ ions in the trap. For a collision energy of $2.2 \mathrm{eV} / \mathrm{amu}$, the $\mathrm{Ar}^{18+}$ extraction data give a hardness ratio of 0.58 , whereas in the $\mathrm{Ar}^{18+}$ magnetic trapping experiment, using a trap depth of $100 \mathrm{~V}$, a hardness ratio approaching double that value, of 1.07 , is obtained. Hardness ratios calculated from the simulated spectra presented in Figure 1 demonstrate that a value of unity corresponds closely to capture into the state $8 s$. Thus the indication is that in the trap electron capture proceeds into even lower $\ell_{c}$ states than is the case for the slowest $\mathrm{Ar}^{18+}$ ions investigated in the extraction experiments. Possible reasons for the discrepancy are now discussed.

In the extraction experiments the projectile energies were measured directly, which is not possible for the HCIs in the trap. Thus a potential cause of the mismatch observed is that the energy of the trapped ions estimated using Equation 3 is incorrect, i.e. it might be an overestimation. However, according to CTMC calculations for capture into $\mathrm{Ar}^{18+}$, hardness ratios of the order of unity and above are not reached [28], even though the maximum hardness ratio from the simulated spectra shown in Figure 1 (corresponding to capture into the $8 p$ state of $\mathrm{Ar}^{18+}$ ) takes a value of $\sim 4$. Instead, for energies decreasing below $1 \mathrm{eV} / \mathrm{amu}$ it is presumed that the hardness 
ratio levels out to a plateau [28]. Consequently, on the basis of the CTMC results an explanation centered on an incorrect assignment of the collision energy in the trap appears to be unlikely. Conclusions based on CTMC results should, however, be made with caution, for the reasons previously outlined.

Turning to differences between the conditions at the external gas target compared to those in the trap, the presence of the $3 \mathrm{~T}$ magnetic field in the latter might be significant. Although a bound electron is not expected to be influenced by such a relatively weak field, since the electrostatic Coulomb attraction of the nucleus is much greater, it is conceivable that once the electron reaches the potential maximum at the critical distance for capture into the ion, the magnetic force could have an effect on its momentum. To test this, magnetic trapping experiments using the EBIT operated at fields down to $1 \mathrm{~T}$ have been carried out, but a change in hardness ratio was not measured. There is of course also the electrostatic field in the trap due to the voltages applied to the drift tubes, but this of the order of $10^{4} \mathrm{~V} / \mathrm{m}$, hence would only result in an electrostatic force on the electron equivalent to the magnetic force just discussed.

A further disparity between the two experimental techniques is that while the HCIs in the trap remain in the viewing range of the x-ray detector for several seconds, the extracted HCIs pass through the viewing range of the $\mathrm{x}$-ray detector in the beamline in a fraction of that time. Therefore it is important to compare the distance traveled by an ion during the radiative cascade, with the viewing length of the detector. Electron cascade times for electric dipole-allowed transitions are of the order of picoseconds, so calculating for the fastest ions $\left(\sim 6.5 \cdot 10^{7} \mathrm{~cm} / \mathrm{s}\right)$, the distance traveled before the cascade ends is of the order of micrometers. With a detector viewing length of $\sim 3.5 \mathrm{~cm}$ it can be concluded that the $K$-shell x-ray photons emitted at the end of such cascades are well within detection range. In contrast, for electron capture into $\mathrm{Ar}^{17+}$ the cascading process efficiently feeds the metastable $1 s 2 s{ }^{1} S_{0}$ (singlet) and $1 s 2 s{ }^{3} S_{1}$ (triplet) states, which have lifetimes of the order of a few hundred nanoseconds [19] corresponding to an ion flight distance of a number of centimeters. Consequently these metastable states, which are thought to receive a considerable proportion of the cascading electrons [19], will decay beyond the range of photon detection. Hence it could be expected that the magnetic trapping hardness ratios for $\mathrm{Ar}^{17+}$, which will include metastable decay, would be lower than the ratios obtained for $\mathrm{Ar}^{17+}$ charge exchange at similar energies in the extraction experiments. In fact, in Figure 4 the magnetic trapping hardness ratios are indeed found to be slightly lower than their extraction counterparts. However, as the real discrepancy is in the results for $\mathrm{Ar}^{18+}$ charge exchange, where the aforemen- tioned metastable states do not arise, an argument based on detector viewing ranges does not clarify the situation either.

\section{CONCLUSIONS}

To summarize, this paper has experimentally determined the energy dependence of $\ell_{c}$ in slow charge exchange collisions over a range of energies previously not investigated. Deviation from a statistical population of $\ell_{c}$ states is confirmed and a rising tendency for capture into $n_{c} p$ states for collision energies decreasing below $\sim 100 \mathrm{eV} / \mathrm{amu}$ is observed.

The cause of the much larger hardness ratios measured for $\mathrm{Ar}^{18+}$ in magnetic trapping mode as opposed to using the extraction setup, however, remains unclarified. The experimental results suggest a difference in the conditions of the EBIT environment compared with those of an external gas target, which in turn leads to a difference in the population mechanism and/or stabilization process of the Rydberg states. It is also possible that the anisotropic x-ray emission from charge exchange at the gas target gives rise to different hardness ratios compared with those measured from the isotropic x-ray emission of the magnetic trapping experiment. The geometry of the gas target experiment presents a similar preferential direction to that of electron beam excitation in an EBIT, for which the anisotropy effect can amount to as much as $20 \%$ [29]. In order to investigate these points detailed theoretical treatment of the problem is required.

In the absence of comparative data to benchmark the magnetic trapping results previously obtained at LLNL [13], it had been concluded that hardness ratio increases much more steeply on decreasing collision energy than the extraction results presented here now reveal. Therefore, charge exchange data obtained using HCIs in the trap should be applied to other scenarios, such as the interpretation of cometary emission, with caution. Furthermore, data analysis has shown that it is also important to compare data collected in magnetic trapping experiments recorded in the same time frame. Experiments using extracted beams of HCIs allow a much more controlled investigation of charge exchange and by incorporating a retardation assembly into the extraction beamline, as in the Berlin setup, charge exchange spectra for a range of collision energies can be obtained. A point to note, however, is that forbidden transitions from the metastable states of HCIs can give rise to intense emissions, as shown by theoretical work in [30]. These intense lines will be absent from the spectra obtained in beamline experiments, though in the case of the relatively large gas densities which can be found in cometary coma, for example, it is likely that such states would be collisionally quenched. 
Charged Ions, edited by F. J. Currell (Dordrecht: Kluwer Academic, 2003), vol. 1, p. 103.

[3] T. Stöhlker, T. Ludziejewski, and H. Reich, Phys. Rev. A 58, 2043 (1998).

[4] C. M. Lisse, K. Dennerl, J. Englhauser, M. Harden, F. E. Marshall, M. J. Mumma, R. Petre, J. P. Pye, M. J. Ricketts, J. Schmitt, et al., Science 274, 205 (1996).

[5] T. E. Cravens, Science 296, 1042 (2002).

[6] H. Ryufuku, K. Sasaki, and T. Watanabe, Phys. Rev. A 21, 745 (1980).

[7] A. Bárány, G. Astner, H. Cederquist, H. Danared, S. Huldt, P. Hvelplund, A. Johnson, H. Knudsen, L. Liljeby, and K.-G. Rensfelt, Nucl. Instr. and Meth. B 9, 397 (1985).

[8] D. Dijkkamp, Y. S. Gordeev, A. Brazuk, A. G. Drentje, and F. J. de Heer, J. Phys. B 18, 737 (1985).

[9] A. Cassimi, S. Duponchel, X. Flechard, P. Jardin, P. Sortais, D. Hennecart, and R. E. Olson, Phys. Rev. Lett. 76, 3679 (1996).

[10] C. M. Lisse, D. Christian, K. Dennerl, J. Englhauser, J. Trmper, M. Desch, F. E. Marshall, R. Petre, and S. Snowden, Icarus 141, 316 (1999).

[11] K. Dennerl, J. Englhauser, and J. Trümper, Science 277, 1625 (1997).

[12] H. Tawara, E. Takács, T. Suta, K. Makónyi, L. P. Ratliff, and J. D. Gillaspy, Phys. Rev. A 73, 012704 (2006).

[13] P. Beiersdorfer, R. E. Olson, G. Brown, H. Chen, C. L. Harris, P. A. Neill, L. Schweikhard, S. B. Utter, and K. Widmann, Phys. Rev. Lett. 85, 5090 (2000).

[14] G. Fussmann, C. Biedermann, and R. Radtke, in Advanced Technologies based on Wave and Beam Generated Plasmas, edited by H. Schlüter and A. Shivarova (Kluwer Academic, Amsterdam, 1999), p. 429.
[15] F. I. Allen, C. Biedermann, R. Radtke, and G. Fussmann, Rev. Sci. Instrum. 77, 03 B903 (2006).

[16] P. Beiersdorfer, L. Schweikhard, J. C. López-Urrutia, and K. Widmann, Rev. Sci. Instrum. 67, 3818 (1996).

[17] S. Fritzsche, J. Electr. Spec. Rel. Phenon. 114-116, 1155 (2001).

[18] S. Fritzsche, Phys. Scr. T100, 37 (2002).

[19] H. Tawara, P. Richard, U. I. Safronova, and P. C. Stancil, Phys. Rev. A 64, 042712 (2001).

[20] A. A. Hasan, F. Eissa, R. Ali, D. R. Schultz, and P. C. Stancil, Astrophys. J. 560, L201 (2001).

[21] J. A. Perez, R. E. Olson, and P. Beiersdorfer, J. Phys. B 34, 3063 (2001).

[22] E. Takács, K. Tőkési, H. Tawara, C. J. Verzani, T. Hohl, J. D. Gillaspy, and J. Pálinkás, Rad. Phys. Chem. 76, 617 (2007).

[23] F. I. Allen, C. Biedermann, R. Radtke, and G. Fussmann, J. Phys. Conf. Series 58, 181 (2007).

[24] F. Currell and G. Fussmann, IEEE Transactions on Plasma Science 33, 1763 (2005).

[25] R. E. Olson, in Springer Handbook of Atomic, Molecular and Optical Physics, edited by G. Drake (Springer New York, 2006), p. 869.

[26] N. Selberg, C. Biedermann, and H. Cederquist, Phys. Rev. A 56, 4623 (1997).

[27] R. Ali, P. A. Neill, P. Beiersdorfer, C. L. Harris, M. J. Raković, J. G. Wang, D. R. Schultz, and P. C. Stancil, Astrophys. J. 629, L125 (2005).

[28] S. Otranto (2006), private communications.

[29] J. Scofield, Phys. Rev. A 40, 3054 (1989).

[30] V. Kharchenko and A. Dalgarno, Astrophys. J. 554, L99 (2001). 